PROCEEDINGS OF THE

AMERICAN MATHEMATICAL SOCIETY

Volume 128, Number 4, Pages 1017-1022

S 0002-9939(99)05134-5

Article electronically published on July 28, 1999

\title{
A NEW CHARACTERISATION OF THE ANALYTIC RADON-NIKODYM PROPERTY
}

\author{
BU SHANGQUAN
}

(Communicated by Dale Alspach)

\begin{abstract}
We show that a separable complex Banach space $X$ has the analytic Radon-Nikodym property if and only if there exists $1 \leq p<\infty$, such that the space consisting of all $L^{p}$-bounded $X$-valued analytic martingales is separable.
\end{abstract}

Let $(X,\|\|$.$) be a complex Banach space and let 1 \leq p \leq \infty . H^{p}(X)$ will denote the space consisting of all analytic functions $f: \mathbf{D} \rightarrow X$ verifying

$$
\|f\|_{p}=\operatorname{Sup}_{0<r<1}\left(\int_{0}^{2 \pi}\left\|f\left(r e^{i \theta}\right)\right\|^{p} \frac{d \theta}{2 \pi}\right)^{1 / p}<\infty
$$

for $1 \leq p<\infty$, and for $p=\infty$

$$
\|f\|_{\infty}=\operatorname{Sup}_{z \in \mathbf{D}}\|f(z)\|<\infty,
$$

where $\mathbf{D}$ is the open unit disk of the complex plane. $H^{p}(X)$ equipped with the norm $\|.\|_{p}$ becomes a Banach space. $X$ is said to have the analytic Radon-Nikodym property (analytic RNP, in short), if there exists $1 \leq p \leq \infty$ (or equivalently for some $1 \leq p \leq \infty)$, such that each $f \in H^{p}(X)$ has radial limits a.e. on $[0,2 \pi]$ in $X$; this means that for almost all $\theta \in[0,2 \pi], \lim _{r \uparrow 1} f\left(r e^{i \theta}\right)$ exists in $X$ (see [1]).

An $X$-valued analytic martingale will be a sequence of integrable functions $f_{n} \in$ $L^{1}\left([0,2 \pi]^{n}, X\right)$ such that $f_{0} \equiv x_{0} \in X$, and for every $n \in \mathbf{N}$, there exists $d_{n} \in$ $L^{1}\left([0,2 \pi]^{n-1}, X\right)$ so that

$$
\begin{gathered}
f_{n}\left(\alpha_{1}, \alpha_{2}, \cdots, \alpha_{n}\right)-f_{n-1}\left(\alpha_{1}, \alpha_{2}, \cdots, \alpha_{n-1}\right) \\
=d_{n}\left(\alpha_{1}, \alpha_{2}, \cdots, \alpha_{n-1}\right) e^{i \alpha_{n}} .
\end{gathered}
$$

It is easy to see that each $X$-valued analytic martingale is an $X$-valued martingale in the usual sense. For $1 \leq p<\infty$, we shall denote by $\mathcal{A}_{p}(X)$ the space of all $L^{p}$-bounded $X$-valued analytic martingales. If $F=\left(f_{n}\right)_{n \geq 0} \in \mathcal{A}_{p}(X)$, define

$$
\|F\|_{p}=\operatorname{Sup}_{n \geq 0}\left\|f_{n}\right\|_{p},
$$

where $\|\cdot\|_{p}$ is a norm on $\mathcal{A}_{p}(X)$ and it is not hard to verify that $\mathcal{A}_{p}(X)$, equipped with the norm $\|\cdot\|_{p}$, becomes a complex Banach space.

Received by the editors May 14, 1998.

1991 Mathematics Subject Classification. Primary 46B20.

Key words and phrases. Analytic Radon-Nikodym property, analytic martingale, separable Banach spaces.

This research was supported by the Natural Sciences Foundation of China and the Fok Ying Tung Education Foundation. 
The analytic RNP has been extensively studied in the last ten years, for instance, it is shown by G.A. Edgar that a complex Banach space has the analytic RNP if and only if there exists $1 \leq p<\infty$, so that each $F=\left(f_{n}\right)_{n \geq 0} \in \mathcal{A}_{p}(X)$ converges in the $L^{p}$-norm (see [2]), and there exists an important relation between $H^{p}(X)$ and $\mathcal{A}_{p}(X)$ (see [3]). For more information about the analytic RNP, we refer to [3], [4], and $[5]$.

M. Daher has established the following elegant characterisation of the analytic RNP for separable complex Banach spaces (see [6]).

Theorem 1. Let $X$ be a separable complex Banach space. $X$ has the analytic $R N P$ if and only if there exists $1 \leq p<\infty$ so that $H^{p}(X)$ is separable.

The purpose of this paper is to establish the analogue of this result in the "analytic martingale" setting. Precisely we shall show the following

Theorem 2. Let $X$ be a separable complex Banach space. $X$ has the analytic $R N P$ if and only if there exists $1 \leq p<\infty$ such that $\mathcal{A}_{p}(X)$ is separable.

Let $X$ be a complex Banach space, $F=\left(f_{n}\right)_{n \geq 0}$ an element of $\mathcal{A}_{p}(X), \quad f_{0} \equiv$ $x_{0} \in X$ and

$$
f_{n}\left(\alpha_{1}, \alpha_{2}, \cdots, \alpha_{n}\right)-f_{n-1}\left(\alpha_{1}, \alpha_{2}, \cdots, \alpha_{n-1}\right)=d_{n}\left(\alpha_{1}, \alpha_{2}, \cdots, \alpha_{n-1}\right) e^{i \alpha_{n}}
$$

For fixed $\left(\theta_{1}, \theta_{2}, \cdots\right) \in[0,2 \pi]^{\mathbf{N}}, G=\left(g_{n}\right)_{n \geq 0}$ defined by $g_{0} \equiv x_{0}$ and

$$
\begin{aligned}
& g_{n}\left(\alpha_{1}, \alpha_{2}, \cdots, \alpha_{n}\right)-g_{n-1}\left(\alpha_{1}, \alpha_{2}, \cdots, \alpha_{n-1}\right) \\
= & d_{n}\left(\theta_{1} \alpha_{1}, \theta_{2}+\alpha_{2}, \cdots, \theta_{n-1}+\alpha_{n-1}\right) e^{i \alpha_{n}} e^{i \theta_{n}}
\end{aligned}
$$

is an $X$-valued analytic martingale and $G=\left(g_{n}\right)_{n \geq 0} \in \mathcal{A}_{p}(X)$. So for each $F=\left(f_{n}\right)_{n \geq 0} \in \mathcal{A}_{p}(X)$, one can define a function from $[0,2 \pi]^{\mathbf{N}}$ into $\mathcal{A}_{p}(X)$ by $S\left(\theta_{1}, \theta_{2}, \cdots\right)=\left(S_{n}\left(\theta_{1}, \theta_{2}, \cdots\right)\right)_{n \geq 0} \in \mathcal{A}_{p}(X)$, by $S_{0} \equiv x_{0}$ and

$$
\begin{gathered}
S_{n}\left(\theta_{1}, \theta_{2}, \cdots\right)\left(\alpha_{1}, \alpha_{2}, \cdots, \alpha_{n}\right)-S_{n-1}\left(\theta_{1}, \theta_{2}, \cdots\right)\left(\alpha_{1}, \alpha_{2}, \cdots, \alpha_{n-1}\right) \\
=d_{n}\left(\theta_{1} \alpha_{1}, \theta_{2}+\alpha_{2}, \cdots, \theta_{n-1}+\alpha_{n-1}\right) e^{i \alpha_{n}} e^{i \theta_{n}} .
\end{gathered}
$$

The proof of Theorem 2 will use the following lemma.

Lemma. Let $X$ be a complex Banach space, $1 \leq p<\infty$, and let $F=\left(f_{n}\right)_{n \geq 0}$ be an element in $\mathcal{A}_{p}(X)$; then $F=\left(f_{n}\right)_{n \geq 0}$ converges in the $L^{p}$-norm if and only if the function $S$ defined above is measurable.

Proof of the Lemma. Let $F=\left(f_{n}\right)_{n \geq 0} \in \mathcal{A}_{p}(X)$; assume that $F=\left(f_{n}\right)_{n \geq 0}$ converges in the $L^{p}$-norm. There exists then $f \in L^{p}\left([0,2 \pi]^{\mathbf{N}}, X\right)$ such that for every $n \in \mathbf{N}$, if $\mathcal{F}_{n}$ is the $\sigma$-algebra on $[0,2 \pi]^{\mathbf{N}}$ generated by the first $n$ coordinates, then $f_{n}=\mathbf{E}\left(f \mid \mathcal{F}_{n}\right)$, where $\mathbf{E}\left(f \mid \mathcal{F}_{n}\right)$ denotes the expectation of $f$ with respect to the $\sigma$-algebra $\mathcal{F}_{n}$. The function

$$
\begin{gathered}
{[0,2 \pi]^{\mathbf{N}} \times[0,2 \pi]^{\mathbf{N}} \rightarrow X,} \\
\left(\left(\alpha_{i}\right)_{i \geq 1},\left(\theta_{i}\right)_{i \geq 1}\right) \rightarrow f\left(\alpha_{1}+\theta_{1}, \alpha_{2}+\theta_{2}, \cdots\right)
\end{gathered}
$$

is clearly measurable and belongs to $L^{p}\left([0,2 \pi]^{\mathbf{N}} \times[0,2 \pi]^{\mathbf{N}}, X\right)$. Hence the function

$$
\begin{gathered}
{[0,2 \pi]^{\mathbf{N}} \rightarrow \mathcal{A}_{p}(X),} \\
\left(\theta_{i}\right)_{i \geq 1} \rightarrow\left(\mathbf{E}\left(f\left(\alpha_{1}+\theta_{1}, \alpha_{2}+\theta_{2}, \cdots\right) \mid \mathcal{F}_{n}\right)\right)_{n \geq 0}
\end{gathered}
$$


is measurable, where the expectation in the expression above is taken for the variables $\left(\alpha_{1}, \alpha_{2}, \cdots\right) \in[0,2 \pi]^{\mathbf{N}}$. But $\left(\mathbf{E}\left(f\left(\alpha_{1}+\theta_{1}, \alpha_{2}+\theta_{2}, \cdots\right) \mid \mathcal{F}_{n}\right)\right)_{n \geq 0}=S\left(\theta_{1}, \theta_{2}, \cdots\right)$; the function $S$ is therefore measurable.

Inversely, assume that the function $S$ is measurable; then for each $\left(\theta_{i}\right)_{i \geq 1} \in$ $[0,2 \pi]^{\mathbf{N}},\left\|S\left(\left(\theta_{i}\right)_{i>1}\right)\right\|_{p}=\|F\|_{p}, S$ is a bounded measurable function. We have $S_{n}=\mathbf{E}\left(S \mid \mathcal{F}_{n}\right)$, where $S_{n}$ is defined by $S_{0} \equiv x_{0} \in \mathcal{A}_{p}(X)$ and for $n \in \mathbf{N}$, $\left(\theta_{1}, \theta_{2}, \cdots, \theta_{n}\right) \in[0,2 \pi]^{n} S_{n}\left(\theta_{1}, \theta_{2}, \cdots, \theta_{n}\right)$ is an $X$-valued analytic martingale which only depends on the first $n$ coordinates and

$$
\begin{gathered}
\left(S_{n}\left(\theta_{1}, \theta_{2}, \cdots, \theta_{n}\right)-S_{n-1}\left(\theta_{1}, \theta_{2}, \cdots, \theta_{n-1}\right)\right)\left(\alpha_{1}, \alpha_{2}, \cdots, \alpha_{n}\right) \\
=d_{n}\left(\theta_{1} \alpha_{1}, \theta_{2}+\alpha_{2}, \cdots, \theta_{n-1}+\alpha_{n-1}\right) e^{i \theta_{n}} e^{i \alpha_{n}} .
\end{gathered}
$$

Indeed, if $S\left(\theta_{1}, \theta_{2}, \cdots\right)=\left(T_{n}\left(\theta_{1}, \theta_{2}, \cdots\right)\right)_{n \geq 0}$, then $T_{0} \equiv x_{0} \in \mathcal{A}_{p}(X)$ and

$$
\begin{aligned}
& \left(T_{n}\left(\theta_{1}, \theta_{2}, \cdots\right)-T_{n-1}\left(\theta_{1}, \theta_{2}, \cdots\right)\right)\left(\alpha_{1}, \alpha_{2}, \cdots, \alpha_{n}\right) \\
& =d_{n}\left(\theta_{1} \alpha_{1}, \theta_{2}+\alpha_{2}, \cdots, \theta_{n-1}+\alpha_{n-1}\right) e^{i \theta_{n}} e^{i \alpha_{n}} .
\end{aligned}
$$

We have to show that for each $A \in \mathcal{F}_{n}$

$$
\int_{A} S(\Theta) d \mu(\Theta)=\int_{A} S_{n}(\Theta) d \mu(\Theta)
$$

where $\Theta=\left(\theta_{1}, \theta_{2}, \cdots\right) \in[0,2 \pi]^{\mathbf{N}}$ and $\mu$ denotes normalized Lebesgue measure on the product space $[0,2 \pi]^{\mathbf{N}}$. Define

$$
\begin{aligned}
Q: \mathcal{A}_{p}(X) & \rightarrow\left(X \times L^{p}([0,2 \pi], X) \times L^{p}\left([0,2 \pi]^{2}, X\right) \times \cdots\right)_{\infty}, \\
G & =\left(g_{n}\right)_{n \geq 0} \rightarrow\left(g_{0}, g_{1}-g_{0}, g_{2}-g_{1}, \cdots\right),
\end{aligned}
$$

where $Q$ is an injective continuous linear application. To show that the equality $(*)$ holds true, it will suffice to show that

$$
Q\left(\int_{A} S(\Theta) d \mu(\Theta)\right)=Q\left(\int_{a} S_{n}(\Theta) d \mu(\Theta)\right)
$$

or equivalently

$$
\int_{A} Q(S(\Theta)) d \mu(\Theta)=\int_{A} Q\left(S_{n}(\Theta)\right) d \mu(\Theta) .
$$

The above equality is an equality between elements in

$$
\left(X \times L^{p}([0,2 \pi], X) \times L^{p}\left([0,2 \pi]^{2}, X\right) \times \cdots\right)_{\infty} .
$$

To show that $(* *)$ holds true, it is sufficient to show that the corresponding coordinates of $\int_{A} Q(S(\Theta)) d \mu(\Theta)$ and $\int_{A} Q\left(S_{n}(\Theta)\right) d \mu(\Theta)$ coincide. Let $Q(S(\Theta))_{(m)}$ be the $m^{\text {th }}$ coordinate of $Q(S(\Theta))$ and let $Q\left(S_{n}(\theta)\right)_{(m)}$ be the $m^{\text {th }}$ coordinate of $Q\left(S_{n}(\Theta)\right)$.

If $1 \leq m \leq n$,

$$
Q(S(\Theta))_{(m)}\left(\alpha_{1}, \alpha_{2}, \cdots, \alpha_{m}\right)=d_{m}\left(\alpha_{1}+\theta_{1}, \alpha_{2}+\theta_{2}, \cdots, \alpha_{m-1}+\theta_{m-1}\right) e^{i \theta_{m}} e^{i \alpha_{m}},
$$$$
Q\left(S_{n}(\Theta)\right)_{(m)}\left(\alpha_{1}, \alpha_{2}, \cdots, \alpha_{m}\right)=d_{m}\left(\alpha_{1}+\theta_{1}, \alpha_{2}+\theta_{2}, \cdots, \alpha_{m-1}+\theta_{m-1}\right) e^{i \theta_{m}} e^{i \alpha_{m}} ;
$$ 
hence

$$
\int_{A} Q(S(\Theta))_{(m)} d \mu(\Theta)=\int_{A} Q\left(S_{n}(\Theta)\right)_{(m)} d \mu(\Theta) .
$$

If $m>n$, then

$$
\begin{gathered}
Q(S(\Theta))_{(m)}\left(\alpha_{1}, \alpha_{2}, \cdots, \alpha_{m}\right)=d_{m}\left(\alpha_{1}+\theta_{1}, \alpha_{2}+\theta_{2}, \cdots, \alpha_{m-1}+\theta_{m-1}\right) e^{i \theta_{m}} e^{i \alpha_{m}} \\
Q\left(S_{n}(\Theta)\right)_{(m)}\left(\alpha_{1}, \alpha_{2}, \cdots, \alpha_{m}\right)=0 .
\end{gathered}
$$

We get

$$
\int_{A} Q(S(\Theta))_{(m)} d \mu(\Theta)=\int_{A} Q\left(S_{n}(\Theta)\right)_{(m)} d \mu(\Theta)=0,
$$

which shows that for every $1 \leq m<\infty$

$$
\int_{A} Q(S(\Theta))_{(m)} d \mu(\Theta)=\int_{A} Q\left(S_{n}(\Theta)\right)_{(m)} d \mu(\Theta),
$$

and hence the equality (**) holds true. As $S_{n}=\mathbf{E}\left(S \mid \mathcal{F}_{n}\right), S_{n}$ converges to $S$ in $L^{p}\left([0,2 \pi]^{n}, \mathcal{A}_{p}(X)\right) . \quad S_{n}$ is then a Cauchy sequence in $L^{p}\left([0,2 \pi]^{\mathbf{N}}, \mathcal{A}_{p}(X)\right)$. For $n, m \in \mathbf{N}$, we have

$$
\begin{gathered}
\left\|S_{n}-S_{n+m}\right\|_{p} \\
=\left(\iint\left\|\sum_{k=n+1}^{n+m} d_{k}\left(\alpha_{1}+\theta_{1}, \cdots, \alpha_{k-1}+\theta_{k-1}\right) e^{i \theta_{k}} e^{i \alpha_{k}}\right\|^{p} d \mu(\Theta) d \mu\left(\alpha_{1}, \alpha_{2}, \cdots\right)\right)^{1 / p} \\
=\left(\int\left\|\sum_{k=n+1}^{n+m} d_{k}\left(\theta_{1}, \theta_{2}, \cdots, \theta_{n-1}\right) e^{i \theta_{k}}\right\|^{p} d \mu(\Theta)\right)^{1 / p} \\
=\left\|f_{n}-f_{n+m}\right\|_{p}
\end{gathered}
$$

hence $F=\left(f_{n}\right)_{n \geq 0}$ is a Cauchy sequence in $L^{p}\left([0,2 \pi]^{\mathbf{N}}, X\right)$ and therefore converges in the $L^{p}$-norm in $X$. This finishes the proof of the Lemma.

Proof of Theorem 2. Suppose that $X$ is separable. If $X$ has the analytic RNP, every $F=\left(f_{n}\right)_{n \geq 0} \in \mathcal{A}_{p}(X)$ converges in the $L^{p}$-norm to an element $f$ of $L^{p}\left([0,2 \pi]^{\mathbf{N}}, X\right)$ and $\|F\|_{p}=\|f\|_{p} \cdot \mathcal{A}_{p}(X)$ is then identified with a closed subspace of $L^{p}\left([0,2 \pi]^{\mathbf{N}}, X\right)$. As $L^{p}\left([0,2 \pi]^{\mathbf{N}}, X\right)$ is separable, $\mathcal{A}_{p}(X)$ is separable.

Inversely, suppose that there exists $1 \leq p<\infty$ such that $\mathcal{A}_{p}(X)$ is separable, and let $F=\left(f_{n}\right)_{n \geq 0} \in \mathcal{A}_{p}(X)$. We have to show that $F$ converges in the $L^{p}$-norm in $X$. By the lemma it is sufficient to show that the function $S$ defined above is measurable.

Let $G=\left(g_{n}\right)_{n \geq 0} \in \mathcal{A}_{p}(X)$ be fixed and $\epsilon>0$; consider the ball

$$
\begin{gathered}
B(G, \epsilon)=\left\{H=\left(h_{n}\right)_{n \geq 0} \in \mathcal{A}_{p}(X):\|H-G\|_{p} \leq \epsilon\right\} \\
=\bigcap_{n \geq 1}\left\{H=\left(h_{n}\right)_{n \geq 0} \in \mathcal{A}_{p}(X):\left\|g_{n}-f_{n}\right\|_{p} \leq \epsilon\right\} .
\end{gathered}
$$


We get

$$
S^{-1}(B(G, \epsilon))=\bigcap_{n \geq 1} S^{-1}\left(\left\{H=\left(h_{n}\right)_{n \geq 0} \in \mathcal{A}_{p}(X):\left\|g_{n}-f_{n}\right\|_{p} \leq \epsilon\right\}\right) .
$$

But for fixed $n \in \mathbf{N}$

$$
\begin{aligned}
& S^{-1}\left(\left\{H=\left(h_{n}\right)_{n \geq 0} \in \mathcal{A}_{p}(X):\left\|g_{n}-h_{n}\right\|_{p} \leq \epsilon\right\}\right) \\
& =\left\{\left(\theta_{1}, \theta_{2}, \cdots\right) \in[0,2 \pi]^{\mathbf{N}}:\left(\int \| g_{n}\left(\alpha_{1}, \alpha_{2}, \cdots, \alpha_{n}\right)\right.\right. \\
& \left.\left.\quad-h_{n}\left(\theta_{1}+\alpha_{1}, \theta_{2}+\alpha_{2}, \cdots, \theta_{n}+\alpha_{n}\right) \|^{p} d \mu\left(\alpha_{1}, \alpha_{2}, \cdots\right)\right)^{1 / p} \leq \epsilon\right\}
\end{aligned}
$$

which is clearly a measurable subset of $[0,2 \pi]^{\mathbf{N}}$; hence $S^{-1}(B(G, \epsilon))$ is a measurable subset of $[0,2 \pi]^{\mathbf{N}}$. As the Borel sets of $\mathcal{A}_{p}(X)$ are generated by balls $\left(\mathcal{A}_{p}(X)\right.$ is separable), the function $S$ is measurable. This finishes the proof.

Let $X$ be a complex Banach space. We shall denote by $H_{0}^{p}([0,2 \pi], X)$ the subspace of $L^{1}([0,2 \pi], X)$ consisting of all $f$, so that the Fourier coefficient $\hat{f}(n)=0$ for a negative integer $n \in \mathbf{Z}$. An $X$-valued integrable sequence $F=\left(f_{n}\right)_{n \geq 0}$ is called an $X$-valued Hardy martingale (see [7]) if $f_{0} \equiv x_{0} \in X$, for each $n \in \mathbf{N}, f_{n} \in$ $L^{1}\left([0,2 \pi]^{n}, X\right)$, and the function $\alpha_{n} \rightarrow f_{n}\left(\alpha_{1}, \alpha_{2}, \cdots, \alpha_{n}\right)-f_{n-1}\left(\alpha_{1}, \alpha_{2}, \cdots, \alpha_{n-1}\right)$ belongs to $H_{0}^{1}([0,2 \pi], X)$ for almost all $\left(\alpha_{1}, \alpha_{2}, \cdots, \alpha_{n-1}\right) \in[0,2 \pi]^{n-1}$. It is easy to see that each analytic martingale is a Hardy martingale and every $X$-valued Hardy martingale is an $X$-valued martingale in the usual sense. $X$ has the analytic RNP if and only if there exists $1 \leq p<\infty$ such that every $L^{p}$-bounded $X$-valued Hardy martingale converges in the $L^{p}$-norm (see [5] and [7]). We denote by $\mathcal{H}_{p}(X)$ the space of all $L^{p}$-bounded $X$-valued Hardy martingales. For $F=\left(f_{n}\right)_{n \geq 0} \in \mathcal{H}_{p}(X)$, define $\|F\|_{p}=\operatorname{Sup}_{n \geq 1}\left\|f_{n}\right\|_{p} ;\|\cdot\|_{p}$ thus defined is a norm on $\mathcal{H}_{p}(X)$. It is not hard to verify that $\mathcal{H}_{p}(X)$ equipped with this norm becomes a Banach space.

Theorem 3. Let $X$ be a separable complex Banach space. $X$ has the analytic $R N P$ if and only if there exists $1 \leq p<\infty$, such that $\mathcal{H}_{p}(X)$ is separable.

Proof of Theorem 3. Let $X$ be a separable complex Banach space. If there exists $1 \leq p<\infty$ such that $\mathcal{H}_{p}(X)$ is separable, as $\mathcal{A}_{p}(X)$ is a closed subspace of $\mathcal{H}_{p}(X), \mathcal{A}_{p}(X)$ is a separable Banach space, by Theorem $2, X$ has the analytic RNP. Inversely, if $X$ has the analytic RNP, then, for every $1 \leq p<\infty$, each $L^{p}$ bounded $X$-valued Hardy martingale converges in the $L^{p}$-norm. For each $F=$ $\left(f_{n}\right)_{n \geq 0} \in \mathcal{H}_{p}(X)$, there exists $f \in L^{p}\left([0,2 \pi]^{\mathbf{N}}, X\right)$ such that $\lim _{n \rightarrow \infty}\left\|f_{n}-f\right\|_{p}=$ $0, f_{n}=\mathbf{E}\left(f \mid \mathcal{F}_{n}\right)$, and $\|f\|_{p}=\|F\|_{p} . \quad \mathcal{H}_{p}(X)$ is then identified with a subspace of $L^{p}\left([0,2 \pi]^{\mathbf{N}}, X\right)$. As $X$ is separable, $L^{p}\left([0,2 \pi]^{\mathbf{N}}, X\right)$ is separable; hence $\mathcal{H}_{p}(X)$ is a separable complex Banach space.

\section{REFERENCES}

[1] A. V. Bukhvalov and A.A. Danilevich, Boundary properties of analytic and harmonic functions with values in Banach spaces, Math. Zametki 31 (1982), 203-214, English translation: Math. Notes, 31 (1982), 104-110. MR 84f:46032

[2] G. A. Edgar, Analytic martingale convergence, J. Funct. Analysis, 69, No. 2 (1986), 268-280. MR 88f:46046

[3] N. Ghoussoub and B. Maurey, Plurisubharmonic martingales and barriers in complex quasiBanach spaces, Ann. Inst. Fourier, 39 (1989), 1007-1060. MR 91g:46003 
[4] N. Ghoussoub, J. Lindenstrauss and B. Maurey, Analytic martingales and plurisubharmonic barriers in complex Banach spaces, Contemp. Math., 85 (1989), 111-130. [MR 90f:46033]

[5] S. Bu and W. Schachermayer, Approximation of Jensen measures by image measures under holomorphic functions and applications, Trans. of A.M.S., 331 (1992), 585-608. MR 92h:46063

[6] M. Daher, Une remarque sur la propriété de Radon-Nikodym, C. R. Acad. Sci. Paris, Tome 313, Serie I (1991), 269-291. MR 92j:46030

[7] B. J. H. Garling, On martingales with values in complex Banach spaces, Math. Pro. Phil. Soc., 104 (1988), 399-406.

Department of Applied Mathematics, Tsinghua University, 100084 Beijing, People's Republic of China

E-mail address: sbu@math.tsinghua.edu.cn 